\title{
Early and Late Pedigree Selection for Seed Yield/Plant in Sesame
}

(Sesamum indicum L.)

\author{
Ismail A.A. ${ }^{1}$; A. Abo-Elwafa ${ }^{1}$; F.S. Sedeck ${ }^{2}$ and A. Abd-Elsaber ${ }^{2}$ \\ ${ }^{1}$ Dept. of Agron., Fac. of Agric. Assiut University \\ ${ }^{2}$ Oil crops Research Section, Field Crops Inst., Agric. Res. Center.
}

\begin{abstract}
:
The present study was carried out at Shandaweel Agricultural Research Station, Sohag Governorate, Egypt during the period of 2009-2011 summer seasons. The means of selected families after two cycles of early selection for seed yield/plant ranged from 31.88 to 43.50 with an average of 37.36 compared to 17.83, 25.47 and $23.33 \mathrm{~g}$ for P1, P2 and bulk sample in population I, respectively. Likewise, these means varied from 29.33 to 39.67 with an average 34.03 compared to their respective parents P1 (19.00) P2 (29.00) and bulk sample (30.0g) in population II. The average of seed yield/plant overall selected families of 41.24 and 42.37 after one cycle of late selection surpassed their averages of 37.36 and $34.03 \mathrm{~g}$ after two cycles of early selection by 10.38 and $24.51 \%$ for population I and II, respectively.

The slight discrepancy between (GCV) and (PCV) resulted in high estimates of broad sense heritability for most studied traits in the two base populations. The selection response to one cycle of late selection for seed yield/plant was large comparing to their values after two cycles of early selection in both populations. The values accounted 61.94 and $76.74 \%$ in population I and 46.11 and $41.24 \%$ in population II with late selection comparing to 46.70 and $60.11 \%$ in population I and 17.36 and $13.44 \%$ in population II as a deviation from the best parent and bulk, respectively.
\end{abstract}

Keywords: sesame, early selection, late selection and seed yield

Received on: 26/10/2014

Accepted for publication on: 19/11/2014

Referees: Prof. Mohamed A. El-Morsidy

Prof. Eman M. Taha 


\section{Introduction:}

Sesame (Sesamum indicum L.) is one of the most ancient cultured oil plants. It has an early origin in East Africa (in ancient Egyptian tombs dating back 4,000 years) and in India (since over 5,000 years ago) (Nayar and Mehra, 1970). Sesame seed is probably the oldest crop grown in China for its taste, dating back 2000 year. The Egyptian used sesame seed as medicine around the same time. The total cultivated area in the world was $66,288,276$ ha produced $4,756,752$ tons, while in Egypt the cultivated area was 30,000 ha produced 40,000 tons of seeds (FAO 2014). In Egypt, there is a large gap between oil production and its consumption. So, sesame cultivars with high seed yield and high content oil are needed. Fortunately sesame is cultivated in hot regions with high solar insulation and tolerates soil droughts (Ustimenko-Bakumovsky, 1983). Consequently, the invention of new varieties of sesame is desirable to grow in Egypt. Holbrook et al. 1989, Pathirana (1995) and El-Shimy (2005) reported that the direct selection for seed yield was the most effective for the improvement of yield in sesame. Ismail et al. (2005) reported that the realized heritability in the two studied populations for seed yield per plant was low compared to that estimated on the basis of mean of the three replications.

Therefore the objective of this study was to estimate the response of pedigree line selection in early and late generations of two sesame ( $\mathrm{Se}$ samum indicum $L$.) populations.

\section{Materials and Methods:}

The present study was carried out at Shandaweel Agricultural Research Station, Sohag Governorate,
Egypt during the period of 2009-2011 summer seasons. The breeding material used in this study was $200 \mathrm{~F}_{3}$ families traced back to random $F_{2}$ plants from each of two crosses i.e. (Introduction 143 x Introduction 245) as population I and (Introduction 520 $\mathrm{x}$ Giza 32) as population II.

In 2009 season, the $200 \mathrm{~F}_{3^{-}}$ families from each population with the original parents, $\mathrm{F}_{3}$-bulked random sample (a mixture of equal number of seeds from each plant to represent the generation mean) were sown on $10^{\text {th }}$ May in two separate experiments in a randomized complete block design with three replications. Each plot was a single row $4 \mathrm{~m}$ long, $55 \mathrm{~cm}$ apart, $10 \mathrm{~cm}$ between hills within a row.

The recommended cultural practices were adopted throughout the growing season. Days to the $50 \%$ flowering for each plot/replication, was recorded. The following traits were measured on ten random plants in each plot; plant height, length of fruiting zone, number of branches/plant, capsule length; number of capsules/plant, seed yield/plant, 1000-seed weight and seed oil percentage which determined by using petroleum either (Bp 40$60^{\circ}$ ) as solvent in soxhalet apparatus according to the method of A.O.A.C 1980.

The first cycle of pedigree line selection (early selection) was applied on the base population for seed yield/plant. The best plant of the best 40 families saved rise the $\mathrm{F}_{4}$ generation.

Season 2010 ( $\mathrm{F}_{4}$ - generation): all the selected and non-selected families for each population, respective parents and the bulk were sown on $13^{\text {th }}$ of May. The same procedures 
and experimental design of the previous season were followed.

Each family was grown in a single row $4 \mathrm{~m}$ long, $55 \mathrm{~cm}$ between rows and $10 \mathrm{~cm}$ between hills. Data were recorded as previously mentioned. The best plant from the best 10 families for seed yield was saved to give the $\mathrm{F}_{5}$ generation.

Season $2011\left(\mathrm{~F}_{5^{-}}\right.$generation):

The same experimental design and field procedure were used to evaluate the two cycles (in $\mathrm{F}_{3}$ and $\mathrm{F}_{4}$ ) of early direct and one cycle of the late direct selection for seed yield/plant $\left(\mathrm{F}_{4}\right)$.

The respective parents and $\mathrm{F}_{5^{-}}$ bulked as random sample were involved in all experiments. Sowing date for all experiments was on $15^{\text {th }}$ of May.

It is of interest to indicate that the comparisons among early and late direct selection was done to detect the effective procedure and the related traits with seed yield/plant in each case.

\section{Statistical Analysis:}

For each season, estimates of phenotypic and genotypic variance and covariance, as well as heritability estimates were calculated from EMS of the variance and covariance components of the selected families.

Data were subjected to proper statistical analysis according to Steel and Torrie (1980). Genotypes means were compared using Revised Least Significant Differences test (RLSD) according to El-Rawi and Khalafala (1980)

- The phenotypic (PCV \%) and genotypic (GCV \%) coefficients of variability were estimated using the formula developed by Burton (1952) as follows:
Phenotypic coefficient of variability:

$$
\operatorname{PCV} \%=\frac{\sigma p}{\bar{X}} \times 100
$$

Genotypic coefficient of variability:

$$
\text { GCV } \%=\frac{\sigma \mathrm{g}}{\overline{\mathrm{X}}} \times 100
$$

Where: $\sigma p$ and $\sigma \mathrm{g}$ are the phenotypic and genotypic standard deviations of the family means, respectively, and $\overline{\mathrm{X}}$ is a family mean for a given trait.

Realized heritability was calculated according to Falconer (1989) from the equation of response $\mathrm{R}=\mathrm{S}$ $\mathrm{h}^{2}$ and the heritability being estimated as the ratio of the $h^{2}=R / S$

where: $\mathrm{R}$ is response to selection, and $\mathrm{S}$ is selection differential.

\section{Results and Discussion:}

\section{I- Description of the base popu- lation:}

The analysis of variance (Table 1) revealed that the $\mathrm{F}_{3}$ - families differed highly significantly for all the studied traits in the two base populations. These results reflect the genetic differences among the $\mathrm{F}_{3}$-families for all the studied characters and could be used for pedigree line selection in the two base populations.

The slight discrepancy between (GCV) and (PCV) (Table 2) resulted in high estimates of broad sense heritability for most studied traits in the two base populations. These data resulted in wide ranges and high estimates of broad sense heritability (more than 56\%) for all studied characters in both base populations, except seed yield / plant (39.51\%) in base population I. 
Table 1: Analysis of variance for the studied traits of the base population $\left(F_{3}\right)$ for population I and II

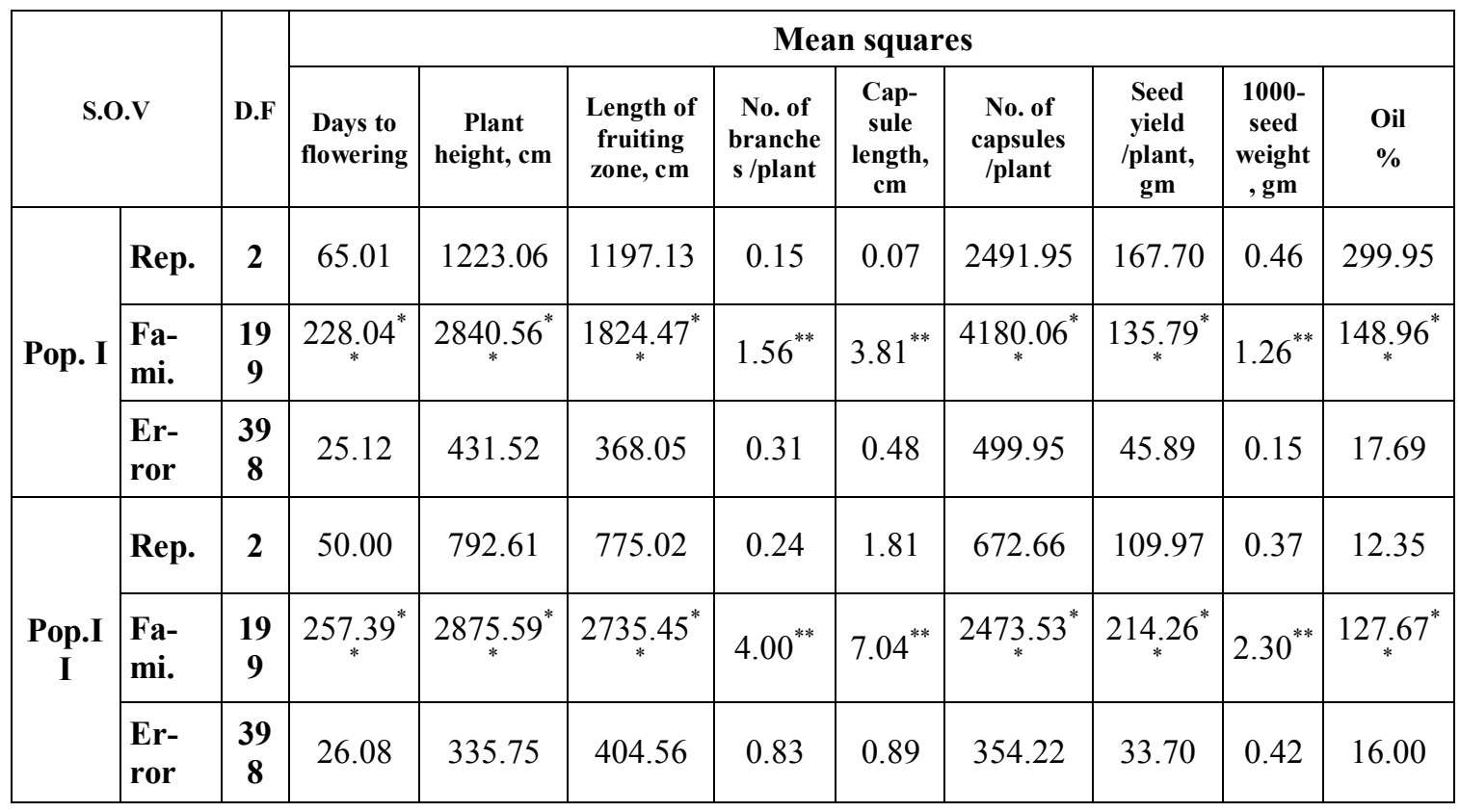

** Significant 0.01 level of probability.

High genotypic and phenotypic variations and heritability estimates for yield and its components were reported by Singh et al (2000), Reddy et al (2001), Saravanan et al (2003), Solanki and Deepak (2003), Singh and Singh (2004), Ganeshan (2005), Mothilal (2006), Supriya (2007), Ganapathy et al (2007), Khan et al
(2007), Prasad et al (2007), Iwo et al (2007), Ghulam et al (2008a), Gangarde et al (2009), Jadhav and Mohrir (2012) and Kumar et al (2012). Otherwise, moderate heritability for plant height, number of capsules, 1000 seed weight and oil was reported by Asha (2005). 
Table 2: Means, phenotypic (PCV \%), genotypic (GCV \%) coefficients of variability and heritability in broad-sense for the studied traits in the two base populations.

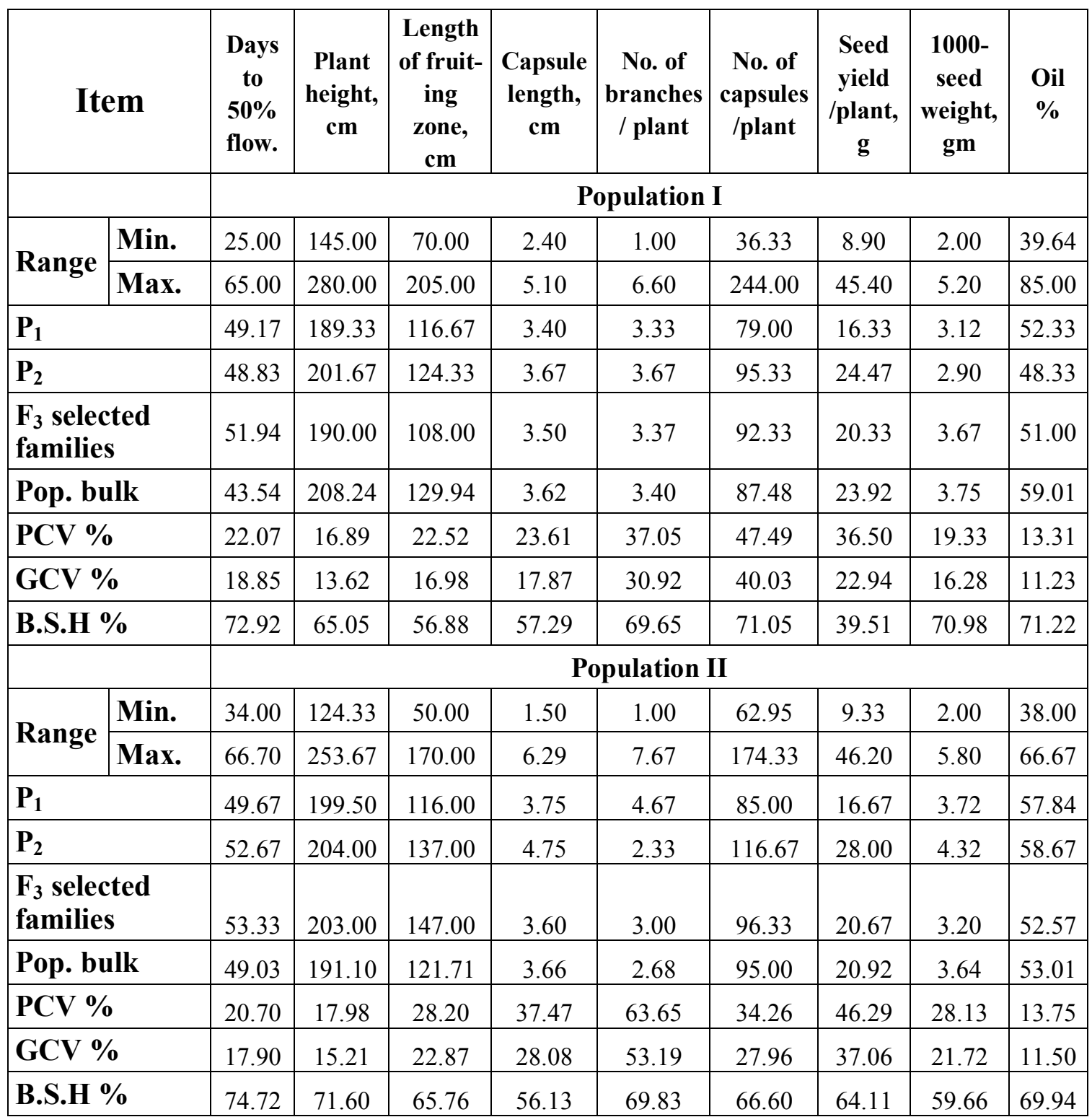

\section{Selection for seed yield/plant}

\subsection{Early pedigree selection}

- Families' mean squares were highly significant in both populations after one and two cycles of early selection for all the studied characters
(Table 3). This result reflects the genetic make-up of those selected families in both populations, indicating that selection could be effective. 
Table 3: Analysis of variance after the first cycle and second cycle of early pedigree selection for seed yield/plant in populations I and 2.

\begin{tabular}{|c|c|c|c|c|c|c|c|c|c|c|c|c|}
\hline & \multirow[b]{2}{*}{ D.F } & \multicolumn{9}{|c|}{ Mean squares } \\
\hline & & & & Days to & $\begin{array}{l}\text { Plant } \\
\text { height }\end{array}$ & \begin{tabular}{|l|} 
Length \\
of fruit-
\end{tabular} & $\begin{array}{c}\text { No. of } \\
\text { branches }\end{array}$ & $\begin{array}{l}\text { Capsule } \\
\text { length. }\end{array}$ & $\begin{array}{c}\text { No. of } \\
\text { capsules }\end{array}$ & $\begin{array}{l}\text { Seed } \\
\text { yield }\end{array}$ & $\begin{array}{c}1000- \\
\text { seed }\end{array}$ & Oil \\
\hline \multirow{6}{*}{ Pop.1 } & \multirow{3}{*}{$\begin{array}{c}\text { C1 } \\
\text { (F4) }\end{array}$} & Rep. & 2 & 53.33 & 129.93 & 96.13 & 0.08 & 0.24 & 107.36 & 0.56 & 0.91 & 2.70 \\
\hline & & Families & 39 & $102.34 * *$ & $1578.01 * *$ & $824.12 * *$ & $0.59 * *$ & $6.19 * *$ & $1838.76^{* *}$ & $48.02 * *$ & $1.45^{* *}$ & $76.90 * *$ \\
\hline & & Error & 78 & 9.23 & 157.38 & 83.59 & 0.06 & 0.57 & 103.13 & 7.15 & 0.19 & 6.67 \\
\hline & \multirow{3}{*}{$\begin{array}{c}\mathrm{C} 2 \\
\text { (F5) }\end{array}$} & Rep. & 2 & 7.82 & 19.66 & 34.36 & 0.04 & 0.58 & 8.25 & 3.68 & 0.01 & 7.99 \\
\hline & & Families & 9 & $87.67 * *$ & $787.09 * *$ & $969.77 * *$ & $0.39 * *$ & $5.87 * *$ & $485.28 * *$ & $43.01 * *$ & $0.77 * *$ & $73.74 * *$ \\
\hline & & \begin{tabular}{|l|} 
Error \\
\end{tabular} & 18 & 4.91 & \begin{tabular}{|l|}
39.35 \\
\end{tabular} & 27.40 & 0.03 & 0.31 & \begin{tabular}{|l|}
25.74 \\
\end{tabular} & 2.10 & 0.03 & 7.19 \\
\hline \multirow{6}{*}{ Pop.1I } & \multirow{3}{*}{$\begin{array}{c}\text { C1 } \\
\text { (F4) }\end{array}$} & Rep. & 2 & 44.10 & 252.41 & 257.56 & 0.22 & 0.42 & 581.93 & 48.84 & 0.48 & 2.45 \\
\hline & & Families & 39 & $73.03 * *$ & $608.00 * *$ & 701.94** & $0.36^{* *}$ & $2.65^{* *}$ & $2165.14 * *$ & $73.45^{* *}$ & $1.89^{* *}$ & $58.60 * *$ \\
\hline & & Error & 78 & 3.28 & 47.54 & 74.04 & 0.03 & 0.25 & 287.99 & 13.97 & 0.20 & 2.57 \\
\hline & \multirow{3}{*}{$\begin{array}{c}\mathrm{C} 2 \\
\text { (F5) }\end{array}$} & Rep. & 2 & 7.43 & 111.70 & 97.23 & 0.38 & 0.16 & 159.11 & 2.63 & 0.06 & 0.64 \\
\hline & & Families & 9 & $47.66 * *$ & $520.83^{* *}$ & $112.07 * *$ & $0.64 * *$ & $1.37 * *$ & $678.82 * *$ & $24.39 * *$ & $1.19^{* *}$ & $18.79 * *$ \\
\hline & & Error & 18 & 2.29 & 26.87 & 5.44 & 0.03 & 0.06 & 29.00 & 1.43 & 0.05 & 0.84 \\
\hline
\end{tabular}

** Significant 0.01 level of probability.

The means of selected families after two cycles of early selection for seed yield/plant (Tables 4 and 5) ranged from $31.88 \mathrm{~g}$ to $43.50 \mathrm{~g}$ with an average of $37.36 \mathrm{~g}$ compared to $17.83 \mathrm{~g}, 25.47 \mathrm{~g}$ and $23.33 \mathrm{~g}$ for $\mathrm{P} 1$, P2 and bulk in population I, respectively. Likewise, these means varied from 29.33 to 39.67 with an average of $34.03 \mathrm{~g}$ compared to their respective parents P1 (19g), P2 (29g) and bulk $(30 \mathrm{~g})$ in population II.

The genotypic coefficients of variation (gcv) for seed yield/plant decreased from 22.94 and $37.06 \%$ in $\mathrm{F}_{3}$ to 9.88 and $8.13 \%$ after two cycles of selection in population I and II, respectively, expressing the increasing of homogeneity in $\mathrm{C}_{2}$ comparing $\mathrm{C}_{1}$.

The phenotypic coefficients of variation (pcv) values were in line with those recorded for $\mathrm{gcv}$ in both populations.

The realized heritability estimated from the realized gain in both cycles of in the two studied populations decreased from $C_{1}$ to $C_{2}$ for the selection criterion of seed yield per plant and all correlated traits, revealing the less genetic variation in $\mathrm{C}_{2}$ comparing to $\mathrm{C}_{1}$. 
Table 4: Mean, range, phenotypic (PCV\%), genotypic (GCV\%) coefficients of variability, heritability in broad-sense (H.B.S) and realized heritability in the two cycles of early pedigree line selection for seed yield/plant in population $I$.

\begin{tabular}{|c|c|c|c|c|c|c|c|c|c|c|c|}
\hline $\begin{array}{c}\text { Cycle } \\
\text { No. }\end{array}$ & \multicolumn{2}{|c|}{ Item } & \begin{tabular}{|c|} 
Days to \\
$50 \%$ \\
flowering
\end{tabular} & $\begin{array}{c}\text { Plant } \\
\text { height, } \\
\mathrm{cm}\end{array}$ & $\begin{array}{l}\text { Length of } \\
\text { fruiting } \\
\text { zone, cm }\end{array}$ & $\begin{array}{c}\text { Capsule } \\
\text { length, } \\
\text { cm }\end{array}$ & $\begin{array}{c}\text { No. of } \\
\text { branches } \\
\text { /plant }\end{array}$ & $\begin{array}{c}\text { No. of } \\
\text { capsules } \\
\text { /plant }\end{array}$ & $\begin{array}{c}\text { Seed } \\
\text { yield } \\
\text { /plant, } \\
\text { g }\end{array}$ & $\begin{array}{c}\text { 1000- } \\
\text { seed } \\
\text { weight, } \\
\text { g }\end{array}$ & $\begin{array}{l}\text { Oil } \\
\%\end{array}$ \\
\hline \multirow{10}{*}{$\begin{array}{c}\mathbf{C}_{1} \\
\left(\mathbf{F}_{4}\right)\end{array}$} & \multirow{2}{*}{ Range } & Min. & 46.33 & 193.67 & 108.33 & 2.97 & 1.33 & 76.00 & 22.86 & 2.81 & 38.00 \\
\hline & & Max. & 68.33 & 276.67 & 186.00 & 4.67 & 7.13 & 196.00 & 41.65 & 5.23 & 61.00 \\
\hline & \multicolumn{2}{|l|}{$\mathbf{P}_{1}$} & 48.00 & 201.17 & 119.00 & 3.40 & 3.33 & 82.33 & 17.33 & 3.12 & 48.00 \\
\hline & \multicolumn{2}{|l|}{$\mathbf{P}_{2}$} & 49.33 & 206.67 & 124.33 & 3.50 & 3.67 & 97.33 & 25.47 & 3.57 & 50.00 \\
\hline & \multicolumn{2}{|l|}{ Bulk } & 54.00 & 230.33 & 136.03 & 3.60 & 3.15 & 98.12 & 22.33 & 3.31 & 52.57 \\
\hline & \multicolumn{2}{|c|}{$\begin{array}{l}F_{4} \text { selected } \\
\text { families }\end{array}$} & 57.46 & 228.05 & 145.94 & 3.84 & 4.23 & 129.68 & 32.27 & 3.92 & 52.82 \\
\hline & \multicolumn{2}{|c|}{ PCV \% } & 11.04 & 11.01 & 12.46 & 12.70 & 36.99 & 20.13 & 14.12 & 20.00 & 10.38 \\
\hline & \multicolumn{2}{|c|}{ GCV \% } & 9.70 & 9.54 & 10.77 & 11.01 & 32.38 & 18.55 & 11.44 & 16.55 & 9.16 \\
\hline & \multicolumn{2}{|c|}{ H.B.S \% } & 77.08 & 75.06 & 74.70 & 75.19 & 76.59 & 84.87 & 65.57 & 68.50 & 77.83 \\
\hline & \multicolumn{2}{|c|}{$\begin{array}{l}\text { Realized } \\
\text { heritability }\end{array}$} & 0.77 & 0.89 & 0.77 & 0.52 & 0.98 & 0.59 & 0.38 & 0.54 & 0.73 \\
\hline \multirow{10}{*}{$\begin{array}{c}C_{2} \\
\left(F_{5}\right)\end{array}$} & \multirow{2}{*}{ Range } & Min. & 51.33 & 227.00 & 146.00 & 3.60 & 1.00 & 133.33 & 31.88 & 3.69 & 44.82 \\
\hline & & Max. & 67.33 & 281.50 & 192.50 & 4.55 & 5.73 & 168.17 & 43.50 & 4.87 & 59.01 \\
\hline & \multicolumn{2}{|l|}{$P_{1}$} & 47.83 & 188.83 & 116.67 & 3.63 & 3.00 & 83.67 & 17.83 & 3.12 & 49.00 \\
\hline & \multicolumn{2}{|l|}{$\mathbf{P}_{2}$} & 50.17 & 204.17 & 121.00 & 3.57 & 3.33 & 95.33 & 25.47 & 3.57 & 51.00 \\
\hline & \multicolumn{2}{|l|}{ Bulk } & 54.67 & 221.67 & 140.00 & 3.70 & 4.00 & 104.67 & 23.33 & 3.97 & 52.57 \\
\hline & \multicolumn{2}{|c|}{$\begin{array}{l}F_{5} \text { selected } \\
\text { families }\end{array}$} & 62.39 & 239.87 & 161.53 & 4.06 & 4.10 & 144.80 & 37.36 & 4.19 & 52.69 \\
\hline & \multicolumn{2}{|c|}{ PCV \% } & 9.14 & 7.08 & 11.44 & 9.41 & 35.85 & 9.24 & 10.62 & 12.46 & 10.29 \\
\hline & \multicolumn{2}{|c|}{ GCV \% } & 8.42 & 6.58 & 10.97 & 8.55 & 33.17 & 8.55 & 9.88 & 11.87 & 8.94 \\
\hline & \multicolumn{2}{|c|}{ H.B.S \% } & 84.90 & 86.36 & 91.98 & 82.59 & 85.64 & 85.61 & 86.66 & 90.74 & 75.52 \\
\hline & \multicolumn{2}{|c|}{$\begin{array}{l}\text { Realized } \\
\text { heritability }\end{array}$} & 0.34 & 0.50 & 0.62 & 0.43 & 0.16 & 0.44 & 0.24 & 0.22 & 0.18 \\
\hline
\end{tabular}


Table 5: Mean, range, phenotypic (PCV\%), genotypic (GCV\%) coefficients of variability, heritability in broad-sense (H.B.S) and realized heritability in the two cycles of early pedigree line selection for seed yield/plant in population II.

\begin{tabular}{|c|c|c|c|c|c|c|c|c|c|c|c|}
\hline $\begin{array}{c}\text { Cycle } \\
\text { No. }\end{array}$ & \multicolumn{2}{|c|}{ Item } & $\begin{array}{c}50 \\
\% \text { Days } \\
\text { to flo- } \\
\text { wering }\end{array}$ & $\begin{array}{c}\text { Plant } \\
\text { height, } \\
\text { cm }\end{array}$ & \begin{tabular}{|c|} 
Length \\
of fruit- \\
ing \\
zone, \\
cm \\
\end{tabular} & $\begin{array}{c}\text { Capsule } \\
\text { length, } \\
\mathrm{cm}\end{array}$ & $\begin{array}{c}\text { No. of } \\
\text { branches } \\
\text { /plant }\end{array}$ & $\begin{array}{c}\text { No. of } \\
\text { capsules } \\
\text { /plant }\end{array}$ & $\begin{array}{c}\text { Seed } \\
\text { yield } \\
\text { /plant, } \\
\text { g }\end{array}$ & $\begin{array}{l}1000- \\
\text { seed } \\
\text { weight, } \\
\mathrm{g}\end{array}$ & $\begin{array}{l}\text { Oil } \\
\%\end{array}$ \\
\hline \multirow{10}{*}{$C_{1}\left(F_{4}\right)$} & \multirow{2}{*}{ Range } & Min. & 45.80 & 207.10 & 109.50 & 3.17 & 1.48 & 57.20 & 14.75 & 2.72 & 44.83 \\
\hline & & Max. & 64.80 & 257.50 & 189.90 & 4.67 & 5.17 & 179.00 & 40.99 & 5.76 & 62.64 \\
\hline & \multicolumn{2}{|l|}{$P_{1}$} & 51.33 & 198.17 & 116.00 & 3.75 & 4.00 & 95.00 & 17.33 & 3.49 & 58.84 \\
\hline & \multicolumn{2}{|l|}{$\mathbf{P}_{2}$} & 53.00 & 199.67 & 123.00 & 4.58 & 3.00 & 104.00 & 29.00 & 4.08 & 59.67 \\
\hline & \multicolumn{2}{|c|}{ Bulk } & 53.67 & 204.18 & 131.67 & 3.80 & 3.03 & 108.28 & 27.00 & 3.51 & 52.57 \\
\hline & \multicolumn{2}{|c|}{$\begin{array}{l}F_{4} \text { selected } \\
\text { families }\end{array}$} & 56.21 & 228.24 & 141.75 & 4.05 & 3.36 & 120.52 & 30.06 & 4.14 & 52.92 \\
\hline & \multicolumn{2}{|c|}{\begin{tabular}{|l|l|} 
PCV \% \\
\end{tabular}} & 9.16 & 6.71 & 11.88 & 9.24 & 30.52 & 25.08 & 19.34 & 21.12 & 8.76 \\
\hline & \multicolumn{2}{|c|}{ GCV \% } & 8.58 & 5.99 & 10.21 & 8.20 & 26.66 & 20.76 & 14.81 & 18.10 & 8.14 \\
\hline & \multicolumn{2}{|c|}{ H.B.S \% } & 87.64 & 79.72 & 73.87 & 78.87 & 76.29 & 68.48 & 58.67 & 73.45 & 86.48 \\
\hline & \multicolumn{2}{|c|}{$\begin{array}{l}\text { Realized heri- } \\
\text { tability }\end{array}$} & 0.84 & 0.72 & 0.78 & 0.45 & 0.87 & 0.51 & 0.38 & 0.26 & -0.87 \\
\hline \multirow{10}{*}{$\begin{array}{c}\mathbf{C}_{2} \\
\left(\mathbf{F}_{5}\right)\end{array}$} & \multirow{2}{*}{ Range } & Min. & 51.50 & 219.50 & 146.00 & 3.80 & 3.20 & 110.00 & 29.33 & 3.60 & 48.18 \\
\hline & & Max. & 65.17 & 257.50 & 167.17 & 5.12 & 5.20 & 164.17 & 39.67 & 5.30 & 55.38 \\
\hline & \multicolumn{2}{|l|}{$\mathbf{P}_{1}$} & 53.33 & 199.50 & 116.00 & 3.58 & 3.97 & 93.00 & 19.00 & 3.78 & 58.84 \\
\hline & \multicolumn{2}{|l|}{$\mathbf{P}_{2}$} & 54.33 & 204.00 & 121.67 & 4.42 & 3.00 & 103.00 & 29.00 & 3.75 & 59.67 \\
\hline & \multicolumn{2}{|c|}{ Bulk } & 54.00 & 223.70 & 123.93 & 3.73 & 3.53 & 106.00 & 30.00 & 3.64 & 52.57 \\
\hline & \multicolumn{2}{|c|}{\begin{tabular}{|l}
$\begin{array}{l}F_{5} \text { selected } \\
\text { families }\end{array}$ \\
\end{tabular}} & 60.87 & 236.00 & 155.63 & 4.40 & 3.90 & 135.42 & 34.03 & 4.29 & 53.14 \\
\hline & \multicolumn{2}{|c|}{ PCV \% } & 6.86 & 5.86 & 4.11 & 11.03 & 18.08 & 11.57 & 8.85 & 15.24 & 4.92 \\
\hline & \multicolumn{2}{|c|}{ GCV \% } & 6.39 & 5.44 & 3.83 & 10.27 & 16.90 & 10.87 & 8.13 & 14.41 & 4.60 \\
\hline & \multicolumn{2}{|c|}{ H.B.S \% } & 86.88 & 85.97 & 86.73 & 86.75 & 87.40 & 88.19 & 84.26 & 89.39 & 87.65 \\
\hline & \multicolumn{2}{|c|}{$\begin{array}{l}\text { Realized heri- } \\
\text { tability }\end{array}$} & 0.45 & 0.39 & 0.32 & 0.43 & 0.64 & 0.28 & 0.18 & 0.25 & 0.53 \\
\hline
\end{tabular}

Realized response of early selection for seed yield/plant

The observed realized response after two cycles of pedigree selection for seed yield/plant were 46.69 and $60.14 \%$ in population I and 17.35 and $13.43 \%$ in population II as measured from the best parent and bulk sample, respectively. Moreover, the highest values of correlated response were recorded for number of capsules/ plant i.e. $51.89 \%$ and $38.35 \%$ in population $I$ and $31.47 \%$ and $27.75 \%$ in population II, followed by length of fruiting zone which revealed 33.50 and 15.38 in population I and 27.92 and 25.58 in population II as a deviation from the best parent and bulk, respectively.

It is clear that the direct and correlated response values of selection were larger in population I than population II in most studied traits. This result exhibited the different genetic make-up of the two current populations.

The superior families after two cycles of selection

The selected families in both populations surpassed significantly 
the respective parents and bulk, except two families (no. 21 and no, 116) in population II. The same trend could be found in correlated traits i.e. plant height, length of fruiting zone and number of capsules / plant in both populations (Tables 6 and 7).

In population 1, the superior family No.153 yielded the highest seed yield/plant $(43.50 \mathrm{~g})$ and surpassed highly significantly the respective parents P1 (17.83g), P2 $(25.47 \mathrm{~g})$ and the bulk sample (23.33g) by $144,70.8$ and $86.5 \%$, respectively. Also, this family (No.153) exceeded significantly the respective parents and the bulk sample in plant height, capsule length, number of branches /plant, number of capsule /plant and 1000-seed weight indicating the strong correlation of those traits with seed yield/plant.

In population II, the superior family No. 47 significantly surpassed the respective parents and bulk sample by $108.08,36.8$ and $32.2 \%$, respectively. This family (No 47) exceeded significantly the respective parents and bulk sample for plant height, Fruiting zone, capsule length, number of capsules/plant and 1000seed weight, explaining the effectiveness' of those traits on seed yield/plant as also recorded in superior family No.153 of population 1 .

It is clear from the obtained results that all families after two cycles of pedigree line selection were late in flowering comparing of their respective parents and bulk in both populations, except the family No. 143 in population II was significantly earlier than its second parent $\left(\mathrm{P}_{2}\right)$ and was early flowering comparing to its first parent $\left(\mathrm{P}_{1}\right)$ and bulk. Moreover, this family surpassed significantly its parents and bulk in seed yield/plant $(34.83 \mathrm{~g})$, plant height $(251.0 \mathrm{~cm})$, length fruiting zone $(163.0 \mathrm{~cm})$, capsule length $(5.12 \mathrm{~cm})$, number of branches/plant (4.53), number of capsules/plant (147.5) and 1000-seed weight $(5.10 \mathrm{~g})$. The obtained results indicated that the pedigree line selection was most efficient procedure for improving seed yield and other companied traits.

2-2. Late pedigree selection for seed yield/plant in the $F_{5}$ generation

The mean squares revealed to be highly significant for seed yield/ plant and all correlated traits, indicating the genetic differences among the selected families in both populations (Table 8).

The average of seed yield/plant overall selected families was 41.24 and $42.37 \mathrm{~g}$ in late selection after one cycle surpassed the average from selected families (37.36 and $34.03 \mathrm{~g}$ ) after two cycles of early selection by 10.38 and $24.51 \%$ for population I and II, respectively. The same trend was found for number of branches and capsules/ plant in both populations and for plant height and fruiting zone in population I as well as 1000 seed weight and oil $\%$ in population II (Table 9).

The overall mean of selected families in late selection was slightly earlier than those of early selection after two cycles of selection by 2.87 to 1.34 days in populations I and II, respectively. Moreover, the overall mean of seed yield/plant for selected families in late selection surpassed their respective parents and bulk sample. 
Table 6: Selected families means after two cycles of early selection for seed yield/plant in population $I$.

\begin{tabular}{|c|c|c|c|c|c|c|c|c|c|}
\hline $\begin{array}{c}\text { Selected } \\
\text { family No. }\end{array}$ & $\begin{array}{c}50 \% \\
\text { Days to } \\
\text { flow. }\end{array}$ & $\begin{array}{c}\text { Plant } \\
\text { height, } \\
\text { cm }\end{array}$ & $\begin{array}{c}\text { Len. of } \\
\text { fruiting } \\
\text { zone, } \mathrm{cm}\end{array}$ & $\begin{array}{c}\text { Capsule } \\
\text { length, } \\
\text { cm }\end{array}$ & $\begin{array}{c}\text { No. of } \\
\text { branches } \\
\text { /plant }\end{array}$ & $\begin{array}{c}\text { No. of } \\
\text { capsules } \\
\text { /plant }\end{array}$ & $\begin{array}{c}\text { Seed } \\
\text { yield } \\
\text { /plant, } \mathrm{g}\end{array}$ & $\begin{array}{c}1000- \\
\text { seed } \\
\text { weight, } g\end{array}$ & $\begin{array}{l}\text { Oil } \\
\%\end{array}$ \\
\hline 48 & 59.23 & 229.50 & 146.50 & 3.85 & 4.20 & 145.67 & 35.00 & 3.69 & 56.30 \\
\hline 56 & 62.33 & 231.00 & 147.83 & 3.60 & 4.20 & 136.27 & 33.30 & 3.84 & 55.32 \\
\hline 88 & 66.33 & 230.50 & 149.50 & 3.60 & 4.70 & 133.33 & 35.17 & 3.79 & 52.25 \\
\hline 106 & 65.33 & 238.00 & 189.33 & 4.25 & 3.00 & 133.72 & 38.25 & 4.87 & 55.15 \\
\hline 111 & 55.33 & 281.50 & 192.50 & 4.55 & 1.00 & 161.33 & 40.00 & 4.87 & 57.81 \\
\hline 115 & 67.33 & 232.50 & 150.67 & 3.85 & 4.50 & 138.10 & 37.00 & 3.74 & 52.25 \\
\hline 122 & 66.33 & 246.00 & 168.50 & 4.35 & 5.50 & 156.97 & 42.50 & 4.26 & 48.00 \\
\hline 126 & 64.33 & 234.67 & 152.00 & 4.10 & 5.00 & 138.00 & 37.00 & 4.29 & 46.00 \\
\hline 135 & 51.33 & 227.00 & 146.00 & 3.85 & 3.20 & 136.47 & 31.88 & 3.74 & 59.01 \\
\hline 153 & 66.00 & 248.00 & 172.50 & 4.55 & 5.73 & 168.17 & 43.50 & 4.84 & 44.82 \\
\hline Mean & 62.39 & 239.87 & 161.53 & 4.06 & 4.10 & 144.80 & 37.36 & 4.19 & 52.69 \\
\hline P1 & 47.83 & 188.83 & 116.67 & 3.63 & 3.00 & 83.67 & 17.83 & 3.12 & 49.00 \\
\hline P2 & 50.17 & 204.17 & 121.00 & 3.57 & 3.33 & 95.33 & 25.47 & 3.57 & 51.00 \\
\hline Bulk & 54.67 & 221.67 & 140.00 & 3.70 & 4.00 & 104.67 & 23.33 & 3.97 & 52.57 \\
\hline RLSD $_{0.05}$ & 3.801 & 10.761 & 8.980 & 0.273 & 0.956 & 8.704 & 2.485 & 0.272 & 4.600 \\
\hline RLSD $_{0.01}$ & 5.206 & 14.741 & 12.301 & 0.374 & 1.310 & 11.923 & 3.404 & 0.373 & 6.301 \\
\hline
\end{tabular}

Table 7: Selected families means after two cycles of early selection for seed yield/plant in population II.

\begin{tabular}{|c|c|c|c|c|c|c|c|c|c|}
\hline $\begin{array}{c}\text { Selected } \\
\text { family } \\
\text { No. }\end{array}$ & $\begin{array}{c}50 \% \\
\text { Days to } \\
\text { flow. }\end{array}$ & $\begin{array}{c}\text { Plant } \\
\text { height, } \\
\text { cm }\end{array}$ & $\begin{array}{l}\begin{array}{c}\text { Len. of } \\
\text { fruiting } \\
\text { zone }\end{array} \\
\end{array}$ & $\begin{array}{l}\text { Capsule } \\
\text { length, } \\
\text { cm }\end{array}$ & $\begin{array}{c}\begin{array}{c}\text { No. of } \\
\text { branches } \\
\text { /plant }\end{array} \\
\end{array}$ & $\begin{array}{c}\text { No. of } \\
\text { capsules } \\
\text { /plant }\end{array}$ & $\begin{array}{c}\text { Seed } \\
\text { yield } \\
\text { /plant, } g\end{array}$ & $\begin{array}{c}1000- \\
\text { seed } \\
\text { weight, } g\end{array}$ & $\begin{array}{l}\text { Oil } \\
\%\end{array}$ \\
\hline 21 & 58.50 & 230.50 & 152.17 & 3.80 & 3.20 & 117.50 & 30.83 & 3.78 & 55.38 \\
\hline 38 & 65.17 & 248.50 & 154.67 & 4.70 & 5.20 & 164.17 & 35.83 & 4.33 & 55.34 \\
\hline 44 & 62.83 & 244.50 & 155.67 & 4.66 & 3.53 & 137.50 & 34.50 & 4.28 & 53.82 \\
\hline 47 & 64.50 & 257.50 & 167.17 & 4.93 & 3.53 & 140.00 & 39.67 & 5.30 & 50.56 \\
\hline 57 & 58.50 & 225.00 & 152.17 & 4.32 & 3.20 & 137.50 & 34.83 & 4.18 & 54.38 \\
\hline 63 & 62.50 & 230.50 & 157.17 & 4.53 & 4.20 & 137.50 & 35.00 & 3.65 & 50.50 \\
\hline 67 & 60.50 & 219.50 & 150.67 & 4.11 & 4.20 & 135.00 & 33.00 & 3.73 & 53.54 \\
\hline 116 & 63.17 & 224.00 & 146.00 & 3.83 & 3.20 & 110.00 & 29.33 & 3.60 & 55.29 \\
\hline 135 & 61.50 & 229.00 & 157.67 & 3.98 & 4.20 & 127.50 & 32.50 & 4.95 & 54.38 \\
\hline 143 & 51.50 & 251.00 & 163.00 & 5.12 & 4.53 & 147.50 & 34.83 & 5.10 & 48.18 \\
\hline Mean & 60.87 & 236.00 & 155.63 & 4.40 & 3.90 & 135.42 & 34.03 & 4.29 & 53.14 \\
\hline P1 & 53.33 & 199.50 & 116.00 & 3.58 & 3.97 & 93.00 & 19.00 & 3.78 & 58.84 \\
\hline P2 & 54.33 & 204.00 & 121.67 & 4.42 & 3.00 & 103.00 & 29.00 & 3.75 & 59.67 \\
\hline Bulk & 54.00 & 223.70 & 123.93 & 3.73 & 3.53 & 106.00 & 30.00 & 3.64 & 52.57 \\
\hline RLSD $_{0.05}$ & 2.59 & 8.89 & 4.00 & 0.30 & 0.43 & 9.24 & 2.05 & 0.37 & 1.57 \\
\hline RLSD $_{0.01}$ & 3.55 & 12.18 & 5.48 & 0.41 & 0.59 & 12.65 & 2.81 & 0.50 & 2.16 \\
\hline
\end{tabular}


Table 8: Analysis of variance after one cycle of late pedigree selection for seed yield/plant in population I and II.

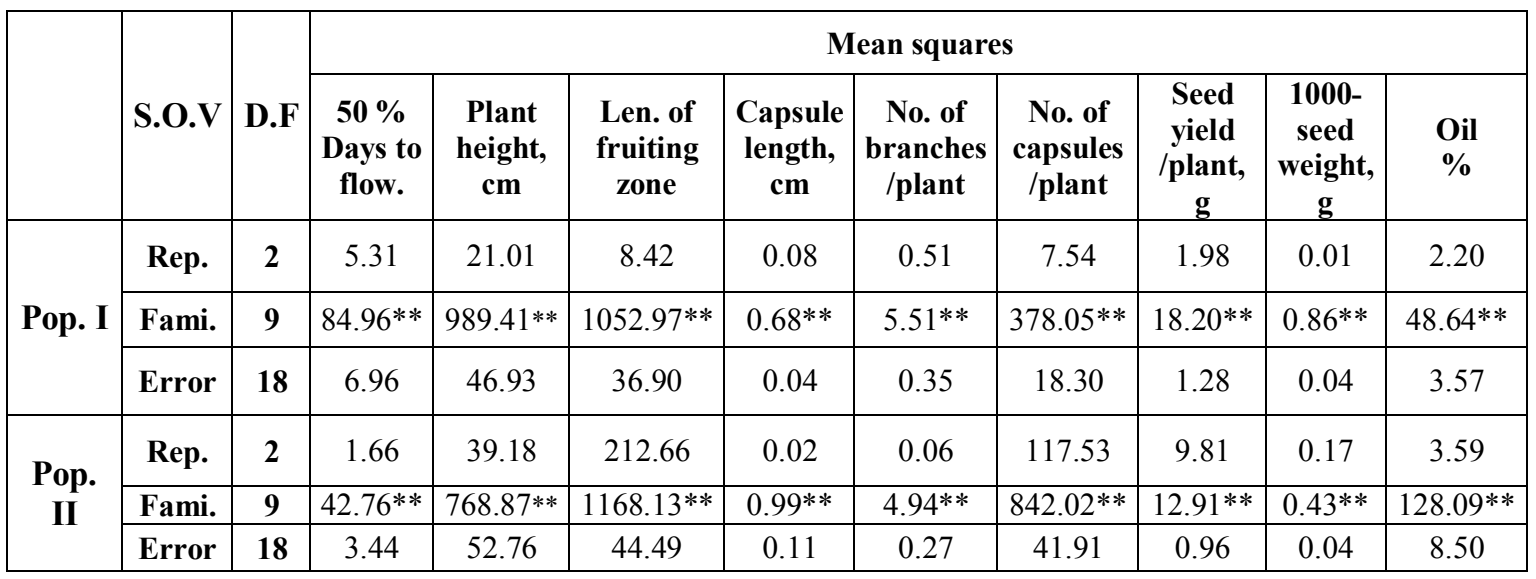

*, ** Significant and highly significant at 0.05 and 0.01 levels of probability, respectively.

Table 9: Means, range, phenotypic (PCV\%), genotypic (GCV\%) coefficients of variability, heritability in broad-sense (H.B.S) and realized heritability in the one cycles of late pedigree selection for seed yield/plant in populations I and II.

\begin{tabular}{|c|c|c|c|c|c|c|c|c|c|c|c|}
\hline & \multicolumn{2}{|c|}{ Item } & $\begin{array}{c}50 \% \\
\text { Days } \\
\text { to } \\
\text { flow. }\end{array}$ & $\begin{array}{c}\text { Plant } \\
\text { height, } \\
\text { cm }\end{array}$ & $\begin{array}{l}\text { Len. of } \\
\text { fruiting } \\
\text { zone }\end{array}$ & $\begin{array}{c}\text { Capsule } \\
\text { length, } \\
\mathrm{cm}\end{array}$ & $\begin{array}{c}\text { No. of } \\
\text { branches } \\
\text { /plant }\end{array}$ & $\begin{array}{c}\text { No. of } \\
\text { capsules } \\
\text { /plant }\end{array}$ & $\begin{array}{c}\text { Seed } \\
\text { yield } \\
\text { /plant, } \\
\text { g } \\
\end{array}$ & $\begin{array}{c}\text { 1000- } \\
\text { seed } \\
\text { weight, } \\
\text { g }\end{array}$ & $\begin{array}{c}\text { Oil } \\
\%\end{array}$ \\
\hline \multirow{10}{*}{ Pop. I } & \multirow{2}{*}{ Range } & Min. & 53.00 & 221.00 & 136.50 & 3.35 & 1.00 & 131.93 & 38.27 & 3.37 & 44.86 \\
\hline & & Maxi. & 66.33 & 281.50 & 198.50 & 4.55 & 5.53 & 166.17 & 44.67 & 4.87 & 57.82 \\
\hline & \multicolumn{2}{|c|}{$\mathbf{P}_{1}$} & 47.83 & 188.83 & 116.67 & 3.63 & 3.00 & 83.67 & 17.33 & 3.12 & 49.00 \\
\hline & \multicolumn{2}{|c|}{$\mathbf{P}_{2}$} & 50.17 & 204.17 & 121.00 & 3.57 & 3.33 & 95.33 & 21.00 & 3.57 & 51.00 \\
\hline & \multicolumn{2}{|c|}{ Bulk } & 54.67 & 221.67 & 140.00 & 3.70 & 4.00 & 104.67 & 23.33 & 3.97 & 52.57 \\
\hline & \multicolumn{2}{|c|}{$F_{5}$ sele. Fami. } & 59.52 & 245.73 & 168.10 & 4.05 & 4.12 & 149.96 & 41.24 & 4.17 & 51.50 \\
\hline & \multicolumn{2}{|c|}{ PCV \% } & 9.64 & 7.73 & 11.53 & 12.40 & 34.89 & 7.84 & 6.54 & 13.38 & 8.37 \\
\hline & \multicolumn{2}{|c|}{ GCV \% } & 8.57 & 7.21 & 10.95 & 11.45 & 31.81 & 7.30 & 5.93 & 12.51 & 7.53 \\
\hline & \multicolumn{2}{|c|}{ H.B.S \% } & 78.89 & 87.00 & 90.18 & 85.30 & 83.13 & 86.76 & 82.34 & 87.43 & 80.78 \\
\hline & \multicolumn{2}{|c|}{ Realized H. } & 0.11 & 0.38 & 0.69 & 0.30 & 0.28 & 0.31 & 0.45 & 0.29 & 0.29 \\
\hline \multirow{10}{*}{$\begin{array}{c}\text { Pop. } \\
\text { II }\end{array}$} & \multirow{2}{*}{ Range } & Min. & 52.33 & 200.50 & 129.50 & 3.50 & 1.73 & 126.00 & 39.80 & 3.53 & 40.23 \\
\hline & & Maxi. & 63.33 & 246.50 & 181.00 & 5.25 & 5.50 & 166.67 & 45.80 & 5.00 & 61.45 \\
\hline & \multicolumn{2}{|c|}{$\mathbf{P}_{1}$} & 53.33 & 199.50 & 116.00 & 3.58 & 3.97 & 93.00 & 19.00 & 3.78 & 58.84 \\
\hline & \multicolumn{2}{|c|}{$\mathbf{P}_{2}$} & 54.33 & 204.00 & 121.67 & 4.42 & 3.00 & 103.00 & 22.67 & 3.75 & 59.67 \\
\hline & \multicolumn{2}{|c|}{ Bulk } & 54.00 & 223.70 & 123.93 & 3.73 & 3.53 & 106.00 & 30.00 & 3.64 & 52.57 \\
\hline & \multicolumn{2}{|c|}{$F_{5}$ sele. Fami. } & 59.53 & 216.20 & 149.72 & 4.22 & 4.09 & 149.05 & 42.37 & 4.47 & 55.61 \\
\hline & \multicolumn{2}{|c|}{ PCV \% } & 6.83 & 7.90 & 13.67 & 15.01 & 33.04 & 11.79 & 5.25 & 9.22 & 12.51 \\
\hline & \multicolumn{2}{|c|}{ GCV \% } & 6.08 & 7.15 & 12.93 & 12.82 & 30.49 & 10.96 & 4.71 & 8.06 & 11.35 \\
\hline & \multicolumn{2}{|c|}{ H.B.S \% } & 79.23 & 81.90 & 89.38 & 72.91 & 85.14 & 86.42 & 80.53 & 76.34 & 82.42 \\
\hline & \multicolumn{2}{|c|}{ Realized H. } & 0.61 & 0.56 & 0.45 & 0.34 & 0.26 & 0.31 & 0.86 & 0.33 & 0.17 \\
\hline
\end{tabular}

by $131.30,61.92$ and $76.77 \%$ in population I and $122.84,89$ and $41.23 \%$ in population II, respectively.

The estimates of PCV, GCV and heritability in broad sense for seed yield/plant were low and accounted 6.54, 5.93 and $82.37 \%$ and $5.25,4.71$ and $80.53 \%$ after one cycle of late selection comparing to $10.62,9.88$ and $86.66 \%$, and 8.85 , 8.13 and $84.26 \%$ after two cycles of early selection in populations I and II, respectively.

High genetic variation as revealed by genotypic and phenotypic variations and heritability for yield 
and its components were reported by Valarmathi et al (2004), Ganeshan (2005), Mothilal (2006), Khan et al (2007), Prasad et al (2007), Iwo et al (2007), Gangarde et al (2009), Jadhav and Mohrir (2012) and Kumar et al (2012). Otherwise, moderate heritability for plant height, number of capsules, 1000 seed weight and oil was reported by Asha (2005). Moreover, low estimates for narrow sense heritability for seed yield / plant and some of its components were reported by Ranganatha et al (1994).

\section{Realized response of late selection for seed yield/plant}

The selection response to one cycle of late selection for seed yield/plant was large comparing to their values after two cycles of early selection in both populations. The values accounted 61.94 and $76.74 \%$ in population I and 46.11 and 41.24 $\%$ in population II with late selection comparing to 46.70 and $60.11 \%$ in population I and 17.36 and $13.44 \%$ in population II as a deviation from the best parent and bulk, respectively.

\section{The superior families after one cycle of late selection}

As mentioned before the average of selected families after one cycle of late selection surpassed those obtained after two cycles of early selection. It is clear that, all selected families surpassed significantly their respective parents and bulk for the criterion seed yield /plant and correlated trait of number of capsules /plant in both populations (Tables 10 and 11).

In population I, the selected families No. 122 and 153 exceeded significantly the best parent and bulk sample in all studied traits, except oil\%. Moreover, the selected family no 111 surpassed the best parent and bulk in all studied traits, except number of branches/ plant. It is importance to mention that those families were matched also the selections of two cycles of early selection in current population.

In population II, the highest family No. 82 in seed yield/plant surpassed significantly the best parent and bulk for all studied traits, except oil $\%$. Same picture of view could be nearly found for selected families No. 13 and 98. Furthermore, the highest selected families for oil \% No. 5, 25 and 109 exceeded significantly the best parent and bulk in oil \%, seed yield/ plant, number of capsules/ plant and 1000-seed weight, as well as length fruiting zone for the former two families (No. 5 and 25).

It is clear that the selected family No. 98 exceed significantly its best parent and bulk for seed yield / plant, number of capsules/ plant, capsule length, length fruiting zone, plant height and 1000-seed weight. In addition to, it was earlier than its parents and bulk sample.

Direct selection for seed yield produced the greater yield response (Holbrook et al. 1989). The pedigree selection line exhibited highest values for seed yield, plant height, number of capsules and 1000-seed weight (Suwan-Jintaanankul 1989). Areeat (1992) noted that the pedigree selection could be used in early generation selection for yield in sesame.

El-Shimy (1995) found that the realized gain for seed yield/plot was $46.34,26.83$ and $21.95 \%$ in one population and 22.95, 36.07 and 40.16 in another population using selection index, independent culling levels and pedigree selection, respectively after two cycles of selection. Moreover, Pathirana (1995) found that the 80 
highest yielding lines recorded a $10.8 \%$ increase in yield compared to the mean of 400 lines.

Baydar et al (1999) found two superior lines had $16.9 \%$ and $15.9 \%$ higher seed yields than the control variety, while one line with $63.25 \%$ oil content was identified as superior for high oil content. Samar et al (2002) reported that selecting for increased yield via selection for number of capsules/plant would be effective.

Abo-Elwafa and Ahmed (2005) reported that significant differences among the $\mathrm{F}_{3}$ families of sesame were recorded for seed yield and its components. The means after two cycles of pedigree line selection presented positive direct response of 25.24 and
$33.03 \%$ and 15.22 and $22.39 \%$ in seed yield/plant over the best parent and the check cultivar for population I and II, respectively. The indirect positive response in seed yield/plant after two cycles of selection yielded 12.62 and $19.63 \%$.

Ismail et al. (2005) reported that the realized heritability in the two studied populations for seed yield/plant was low compared to that estimated on the basis of mean of the three replications. Moreover, three cycles of pedigree selection increased seed yield by 28.64 and $31.53 \%$ from the bulk sample in populations I and II, respectively.

Table 10: Selected families means after one cycle of late selection for seed yield/plant in population $I$.

\begin{tabular}{|c|c|c|c|c|c|c|c|c|c|}
\hline $\begin{array}{c}\text { Selected } \\
\text { family } \\
\text { No. }\end{array}$ & $\begin{array}{c}50 \% \\
\text { Days to } \\
\text { flow. }\end{array}$ & $\begin{array}{c}\text { Plant } \\
\text { height, } \\
\text { cm }\end{array}$ & $\begin{array}{l}\text { Length of } \\
\text { fruiting } \\
\text { zone, } \mathrm{cm}\end{array}$ & $\begin{array}{l}\text { Capsule } \\
\text { length, } \\
\text { cm }\end{array}$ & $\begin{array}{c}\begin{array}{c}\text { No. of } \\
\text { branches } \\
\text { /plant }\end{array} \\
\end{array}$ & $\begin{array}{c}\text { No. of } \\
\text { capsules } \\
\text { /plant }\end{array}$ & $\begin{array}{c}\text { Seed } \\
\text { yield } \\
\text { /plant, } \mathrm{g}\end{array}$ & $\begin{array}{c}1000- \\
\text { seed } \\
\text { weight, } g\end{array}$ & $\begin{array}{l}\text { Oil } \\
\%\end{array}$ \\
\hline 4 & 65.67 & 270.50 & 181.50 & 4.45 & 4.80 & 152.97 & 44.67 & 4.07 & 49.06 \\
\hline 32 & 55.00 & 229.50 & 150.50 & 5.45 & 3.53 & 133.47 & 38.27 & 3.37 & 55.00 \\
\hline 40 & 59.00 & 236.50 & 159.50 & 3.90 & 3.93 & 149.00 & 39.67 & 4.17 & 50.00 \\
\hline 42 & 63.67 & 243.50 & 178.50 & 3.85 & 4.37 & 152.97 & 43.67 & 4.47 & 52.94 \\
\hline 52 & 55.23 & 245.33 & 184.00 & 4.45 & 5.33 & 159.67 & 43.00 & 4.52 & 48.59 \\
\hline 111 & 55.33 & 281.50 & 198.50 & 4.55 & 1.00 & 161.33 & 40.00 & 4.87 & 57.82 \\
\hline 122 & 66.33 & 246.00 & 168.50 & 4.35 & 5.50 & 146.97 & 42.50 & 4.26 & 48.00 \\
\hline 153 & 66.00 & 248.00 & 172.50 & 4.55 & 5.53 & 166.17 & 43.50 & 4.84 & 44.86 \\
\hline 164 & 53.00 & 221.00 & 136.50 & 3.35 & 3.53 & 131.93 & 38.47 & 3.37 & 55.36 \\
\hline 177 & 56.00 & 235.50 & 151.00 & 3.60 & 3.70 & 145.17 & 38.67 & 3.82 & 53.42 \\
\hline Mean & 59.52 & 245.73 & 168.10 & 4.05 & 4.12 & 149.96 & 41.24 & 4.17 & 51.50 \\
\hline P1 & 47.83 & 188.83 & 116.67 & 3.63 & 3.00 & 83.67 & 17.83 & 3.12 & 49.00 \\
\hline P2 & 50.17 & 204.17 & 121.00 & 3.57 & 3.33 & 95.33 & 25.47 & 3.57 & 51.00 \\
\hline Bulk & 54.67 & 221.67 & 140.00 & 3.70 & 4.00 & 104.67 & 23.33 & 3.97 & 52.57 \\
\hline RLSD $_{0.05}$ & 4.52 & 11.75 & 10.42 & 0.33 & 1.01 & 7.34 & 1.94 & 0.34 & 3.24 \\
\hline $\operatorname{RLSD}_{0.01}$ & 6.20 & 16.10 & 14.27 & 0.45 & 1.39 & 10.05 & 2.66 & 0.47 & 4.44 \\
\hline
\end{tabular}


Table 11: Selected families means after one cycle of late selection for seed yield/plant in population II.

\begin{tabular}{|c|c|c|c|c|c|c|c|c|c|}
\hline $\begin{array}{c}\text { Selected } \\
\text { family } \\
\text { No. } \\
\end{array}$ & $\begin{array}{c}50 \% \\
\text { Days to } \\
\text { flow. } \\
\end{array}$ & $\begin{array}{c}\text { Plant } \\
\text { height, } \\
\text { cm }\end{array}$ & $\begin{array}{c}\text { Length of } \\
\text { fruiting } \\
\text { zone, } \mathrm{cm} \\
\end{array}$ & $\begin{array}{c}\text { Capsule } \\
\text { length, } \\
\text { cm }\end{array}$ & $\begin{array}{c}\begin{array}{c}\text { No. of } \\
\text { branches } \\
\text { /plant }\end{array} \\
\end{array}$ & $\begin{array}{l}\text { No. of } \\
\text { capsules } \\
\text { /plant } \\
\end{array}$ & $\begin{array}{c}\text { Seed } \\
\text { yield } \\
\text { /plant, } g\end{array}$ & $\begin{array}{c}1000- \\
\text { seed } \\
\text { weight, } g\end{array}$ & $\begin{array}{l}\text { Oil } \\
\%\end{array}$ \\
\hline 5 & 60.33 & 212.00 & 138.50 & 4.27 & 4.00 & 165.00 & 42.00 & 4.50 & 61.45 \\
\hline 13 & 63.33 & 20.00 & & & 5.50 & 166.67 & 45.33 & 4.60 & 49.57 \\
\hline 25 & 62.33 & 211.00 & 143.00 & 4.00 & 3.00 & 135.50 & 41.50 & 4.70 & 60.00 \\
\hline 45 & 62.33 & 216.50 & 137.00 & 4.00 & 4.67 & 166.67 & 43.80 & 4.48 & 56.78 \\
\hline 82 & 63.00 & 242.00 & 181.00 & 5.00 & 5.50 & 165.00 & 45.80 & 5.00 & 40.23 \\
\hline 98 & 52.33 & 246.50 & 177.67 & 5.25 & 1.73 & 137.00 & 43.00 & 4.65 & 57.00 \\
\hline 109 & 55.33 & 202.50 & 129.50 & 3.75 & 5.00 & 152.50 & 40.00 & 4.33 & 61.31 \\
\hline 110 & 56.00 & 206.00 & 173.00 & 3.50 & 2.67 & 126.00 & 41.50 & 4.45 & 53.81 \\
\hline 119 & 59.00 & 200.50 & 132.50 & 3.75 & 3.83 & 126.17 & 39.80 & 3.53 & 56.00 \\
\hline 136 & 61.33 & 205.00 & 137.00 & 4.03 & 5.00 & 150.00 & 41.00 & 4.40 & 59.93 \\
\hline Mean & 59.53 & 216.20 & 149.72 & 4.22 & 4.09 & 149.05 & 42.37 & 4.47 & 55.61 \\
\hline P1 & 53.33 & 199.50 & 116.00 & 3.58 & 3.97 & 93.00 & 19.00 & 3.78 & 58.84 \\
\hline P2 & 54.33 & 204.00 & 121.67 & 4.42 & 3.00 & 103.00 & 29.00 & 3.75 & 59.67 \\
\hline Bulk & 54.00 & 223.70 & 123.93 & 3.73 & 3.53 & 106.00 & 30.00 & 3.64 & 52.57 \\
\hline RLSD $_{0.05}$ & 3.18 & 12.46 & 11.44 & 0.57 & 0.89 & 11.11 & 1.68 & 0.34 & 5.00 \\
\hline & 4.36 & 17.07 & 15.67 & 0.78 & 1.22 & 15.21 & 2.31 & 0.47 & 6.85 \\
\hline
\end{tabular}

\section{References:}

A.O.A.C. 1980. Official Methods of Analysis Association of Official Agric. Chemists $13^{\text {th }}$ ed. A.O.A.C., Washington, D.C.

Abo-Elwafa, A. and T. A. Ahmed 2005. Efficiency of line selection and contributions of different traits in seed yield and oil through two cycles of selection in sesame (Sesamum indicum L.). Assiut Journal of Agricultural Sciences. 2005. 36: 2, 124. 24 ref.

Areeat, S.N. 1992. Evaluation of early generation selection procedures for yield in sesame ( $\mathrm{Se}$ samum indicum L.). Sesame and Safflower Newsletter, 7: 112113.

Asha, M.B., 2005. Genetic analysis of induced variability for quantitative traits in sesame (Sesamum indicum L.). M.Sc.(Agri.)
Thesis, Univ. Agric. Sci., Dharwad.

Baydar, H.; R. Marqurad and I. Turgut 1999. Pure line selection for improved yield, oil content and different fatty acid composition of sesame (Sesamum indicum L.). Plant Breeding. 118: 462464.

Burton, G.W. 1952. Quantitative inheritance in grasses. $6^{\text {th }}$ Internat. Grassland Cong. Proc. 1: 273-283.

El-Rawi, K. and A. M. Khalafala, 1980. Design and Analysis of Agricultural Experiments. ElMousel Univ. Iraq.

El-Shimy, A. A., 2005, Contributions of different traits in seed yield through two cycles of line selection in sesame (Sesamum indicum L.). Assiut Journal of Agricultural Sciences. 2005. 36: 4, 125-141. 25 ref. 
FAO,2014.Http://appsl.fao.org/servle t/xte.servlet.jrun.

Falconer, D.S. 1989. Introduction to Quantitative Genetics. Hong Kong, London.

Ganapathy, S.; S. K. Ganesh; P. Vivekanandan and $\mathrm{K}$. B. Kumar, 2007. Variability, heritability and genetic advance in sesame (Sesamum indicum L.). Research on Crops. 2007. 8: 3, 641-643. 5 ref. India

Ganeshan, K. N. 2005. Variability studies in determinate type sesame, Sesamum indicum L. germplasm lines for yield and its component traits. J. Oilseeds Res., 22(1): 176- 177.

Gangarde, S. A.; N. D. Bangar and T. D. Katore, 2009. Variability, heritability and genetic advance in sesame (Sesamum indicum L.). Journal of Maharashtra Agricultural Universities. 2009. 34: 2, 147-148. 7 ref.

Ghulam, S.; M.A. Haq; A. C. Akbar and M.B. Chaudhary, 2008. Induced polygenic variation study in sesame (Sesamum indicum L.), and its implication in selection induced and senra. Academic Publishers, Burnaby, British Columbia Vol. 2, No. 2, pp. 399-403, 2008

Holbrook, C.C.; J.W. Buton and T.E.J.R. Carter 1989. Evaluation of recurrent restricted index selection for increasing yield while holding seed protein constant in soybean. Crop Sci., 29: 324-329.

Ismail, A.A., B.R. Bakheit; A.A. ElShimy and F.S. Sedeck, 2005. Pedigree selection and independent culling levels methods in sesame (Sesamum indicum L.). The conference of agronomy,
Agron. Dept., Fac. Agric. Assiut Univ., Nov. 15-16, 2005, (443459).

Iwo, G. A.; A. A. Idowu and S. Misari, 2007. Genetic variability and correlation studies in sesame (Sesamum indicum L.). Global Journal of Pure and Applied Sciences (Nigeria). 2007. 13: 1, 35-38. 21 ref.

Jadhav, R. S. and M. N. Mohrir, 2012. Genetic variability studies for quantitative traits in sesame (Sesamum indicum L.). Electronic Journal of Plant Breeding (Indian); 2012. 3: 4, 1009-1011. 10 ref.

Khan, M. A.; M. Y Mirza; M. Akmal; Naazar Ali and Ihsanullah Khan, 2007. Genetic parameters and their implications for yield improvement in sesame. Sarhad Journal of Agriculture. 2007. 23: 3, 623-627. 19 ref.

Kumar, S.; R. R. Gupta; R. Chandra and G. R. Gupta, 2012. Selection parameters for high yield and oil content in sesame ( $\mathrm{Se}$ samum indicum L.). Current Advances in Agricultural Sciences(Indian); 2012. 4: 2, 156-158. 10 ref.

Mothilal, A., 2006. Genetic variability and correlation studies for yield and its component characters in sesame (Sesamum indicum). Journal of Ecobiology. 2006. 18: 2, 117-120. 10 ref.

Nayar, N. M. and K. L. Mehra. 1970. Sesame its uses, botany, cytogenetic and origin. Eco. Bot. 24 (1) : 20-31.

Pathirana, R. 1995. Comparison of selection procedures in breeding for seed yield in segregating sesame populations. Euphytica. 82: 73-78. 
Prasad, Y. V. N. S.; N. D. Bangar and P. A. Navale, 2007. Genetic variability, correlation and path analysis in F (2) and F (3) population of cross JLSV 4 x Hawari in sesamum. Journal of Maharashtra Agricultural Universities. 2007. 32: 1, 59-61. 11 ref.

Ranganatha, A.R.G.; K. Virupakshappa; T. Srinives and G. Shivashankar 1994. Effectiveness of early generation yield testing in sesame. Directorate of Oilseeds Research, Hyderabad, India. Oileaginex. 49: 55-58.

Reddy, P. A. V.; M. R. Sekhar; A. R. G. Ranganatha and A. Dhanraj 2001. Genetic variability and heritability for seed yield and its components in sesame (Sesamum indicum L.). J. Oilseeds Res., 18 (2): 173-175.

Samar, A.M.; El-Shakhess; M.A. Madkour and M.G.M. El-Bas 2002. Yield Improvement in Sesame (Sesamum indicum L.) via selection index. Egypt J. Plant Breed. 6 (2): 121-132.

Saravanan, S.; N. Nadarajan and R. U. Kumari 2003. Variability studies in sesame. Crop Res., Hissar, 25(2): 325-327.

Singh, H.C.; V.P. Nagaich and S.K. Singh 2000. Genetic variability for dry matter production in sesame (Sesamum indicum L.). Annals of Agric. Research. 21: 323-327.
Singh, S.B. and S. D. Singh 2004. Estimation of variability parameters for some quantitative characters in sesame (Sesamum indicum). Ann. Agric. Res., 25(1): 43-45.

Solanki, Z.S. and G. Deepak 2003. Variability and character association among quantitative characters of sesame. J. Oilseeds Res., 20(2): 276-277.

Steel, R.G.D. and J.H. Torrie 1980. The principles and procedures of statistics: A biometrical a proch. Mc. Craw-Hill Book Co., New Yourk, USA.

Supriya, K. 2007. Genetic variability studies in identified mutants of sesame (Sesamum indicum L.). M. S. Univ. Agric Sci Dharwad.

Suwan-Jintaanankul 1989. Comparison of selection methods in sesame (Sesamum indicum L.) Bangkok (Thailand) 94 leaves. (C.F. Computer Search International Agric. Cent. for Information Service).

Ustimenko-Bakumovsky, G.V. 1983. Plant growing in the tropics and subtropics. Pp.392, Mir publishers, Moscow.

Valarmathi, G.; M. Kumar and N. A. Saravanan, 2004.Genetic variability and correlation studies for seed related traits in sesame. Sesame and Safflower Newsletter. 2004. 19, unpaginated. 


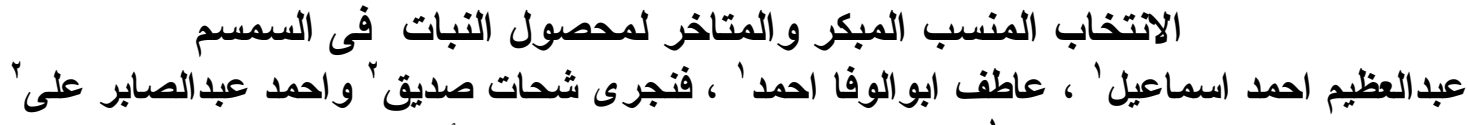

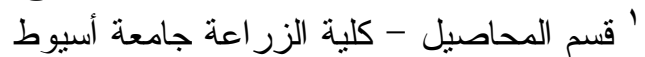

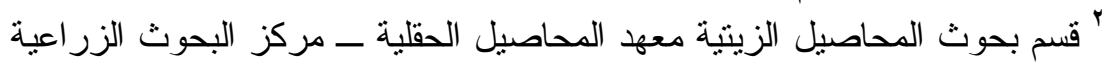

أجريت هذه الدر اسة في مزر عة محطة بحوث جزيرة شندويل بمحافظة ســوهاج التابعــة

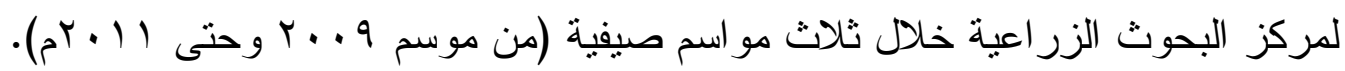

كان متوسط محصول العائلات المنتخبة بعد دورتين من الانتخاب المبكر يتر اوح ما بـين

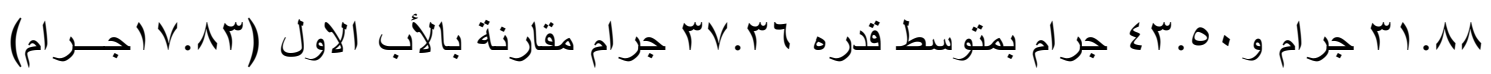

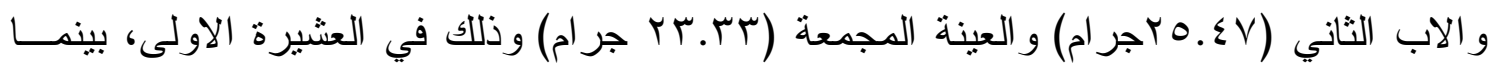

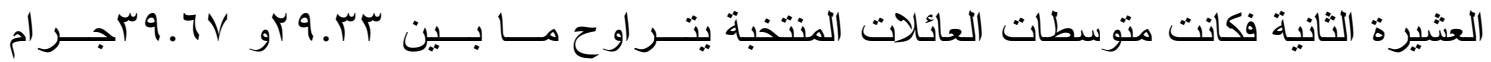

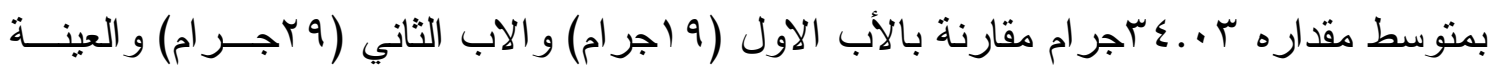
المجمعة ( • ج جر ام). وكان متوسط محصول العائلات المنتخبة بعد دورة من الانتخاب المتــأخر

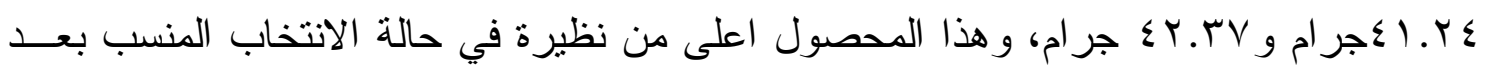

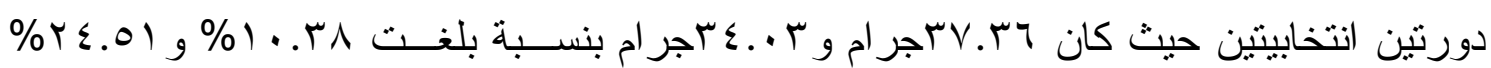
للعثيرة الاولى و الثانية على التو الي.

وجد اختلاف طفيف بين (GCV) و (PCV) مع وجود تقدير ات عالية من درجة التوريث

بالمعنى الو اسع لمعظم الصفات المدروسة في عشيرتيا الاساس. الاستجابة المرتبطــــة للانتخــاب بعد دورة واحدة من الانتخاب المتأخر لمحصول البذور للنبات أكبر من الاستجابة المرتبطة بعـد

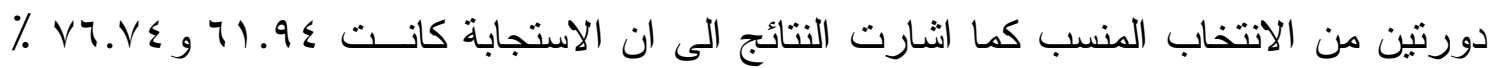

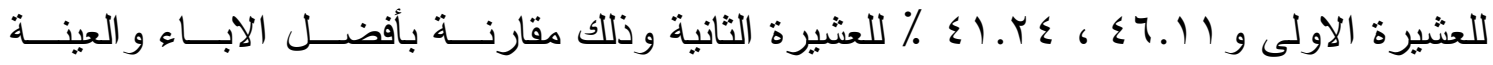
المجمعة على التو الي. 